\title{
First Results of the PixelGEM Central Tracking System for COMPASS
}

\author{
Alexander Austregesilo ${ }^{\mathrm{a}}$, Florian Haas ${ }^{\mathrm{a}}$, Bernhard Ketzer ${ }^{\mathrm{a}}$, Igor Konorov ${ }^{\mathrm{a}}$, Markus Krämer ${ }^{\mathrm{a}}$, \\ Alexander Mann ${ }^{\mathrm{a}}$, Thiemo Nagel ${ }^{\mathrm{a}}$, Stephan Paul ${ }^{\mathrm{a}}$ and Sebastian Uhl ${ }^{\mathrm{a}}$ \\ atechnische Universität München, Physik Department E18 \\ James-Franck-Straße, 85748 Garching, Germany
}

For its physics program with a high-intensity hadron beam of up to $2 \cdot 10^{7}$ particles/s, the COMPASS experiment at CERN requires tracking of charged particles scattered by very small angles with respect to the incident beam direction. While good resolution in time and space is mandatory, the challenge is imposed by the high beam intensity, requiring radiation-hard detectors which add very little material to the beam path in order to minimize secondary interactions.

To this end, a set of triple-GEM detectors with a hybrid readout structure consisting of pixels in the beam region and 2-D strips in the periphery was designed and built. Successful prototype tests proved the performance of this new detector type, showing both extraordinary high rate capability and detection efficiency. The amplitude information allowed to achieve spatial resolutions about a factor of 10 smaller than the pitch and a time resolution close to the theoretical limit imposed by the layout.

The PixelGEM central tracking system consisting of five detectors, slightly improved with respect to the prototype, was completely installed in the COMPASS spectrometer in spring 2008.

\section{Introduction}

COMPASS (COmmon Muon and Proton Apparatus for Structure and Spectroscopy) is a twostage magnetic spectrometer, built for the investigation of the gluonic and quarkonic structure of nucleons and the spectroscopy of hadrons using high-intensity muon and hadron beams from CERN's Super Proton Synchrotron (SPS) [1].

Since spring 2008, hadron beams of up to $2 \cdot 10^{7}$ particles/s are used to perform spectroscopy of mesons and baryons in the light quark sector. These channels require the tracking of charged particles scattered by very small angles with respect to the incident beam, calling for detectors with good resolution in space and time in order to disentangle pile-up and multi-track events within

\footnotetext{
*CERN, 1211 Genve 23, Switzerland, phone: +41 22 7676466, mail: Alexander.Austregesilo@cern.ch

We gratefully acknowledge the skills of Rui de Oliveira and his group at CERN TS-DEM-PMT, and the support of Christian Joram, Eric David, Miranda van Stenis, and Ian McGill of CERN PH-DT2. We are also grateful to Panasonic Electric Works Deutschland $\mathrm{GmbH}$ for providing us with samples of high-density connectors.
}

the primary beam. Further demands of the high hadron flux density are radiation hardness and minimal material budget in order to avoid secondary interactions. Since the beginning of the 2008 beam time, this task is performed by a set of five newly developed triple-GEM [2, 3] beam trackers with a hybrid readout structure, consisting of pixels in the central region and strips in two orthogonal projections in the periphery. Within the spectrometer, the so called PixelGEM detectors are grouped into three stations, two of the latter consisting of two specimen which are rotated by $\pi / 4$ against each other. Owing to the redundancy given by the silicon vertex tracking detectors, the first station upstream of the first analyzing magnet consists of only one PixelGEM detector to minimize interactions there.

\section{The PixelGEM detector}

The amplification stage of the PixelGEM detector uses the geometry already chosen for the COMPASS triple-GEM detectors with strip readout. This setup consists of a $3 \mathrm{~mm}$ drift gap and three GEM foils, separated by $2 \mathrm{~mm}$ transfer gaps 


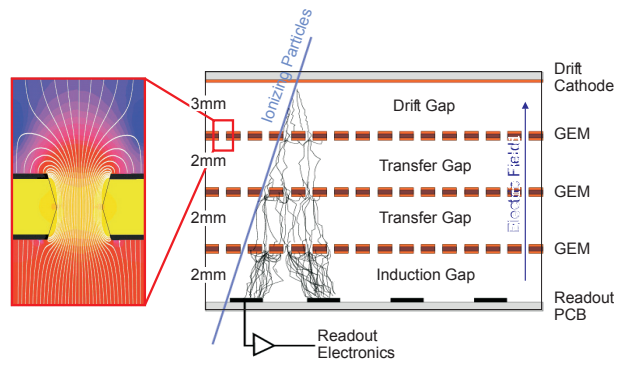

Figure 1. Schematic view of a GEM setup

from each other and by a $2 \mathrm{~mm}$ induction gap from the readout circuit (c.f. Figure 1).

Avalanche charge amplification takes place in microscopic holes with a diameter of $70 \mu \mathrm{m}$, which are placed in a hexagonal pattern with a pitch of $140 \mu \mathrm{m}$ on a $50 \mu \mathrm{m}$ thick polyimide foil, coated with copper on both sides.

The readout structure has been realized on a polyimide printed circuit foil of only $100 \mu \mathrm{m}$ total thickness with three conductive layers. The pixel size has been chosen to be $1 \times 1 \mathrm{~mm}^{2}$, which constitutes a compromise between spatial resolution achievable and number of channels. Thus, the central area of $32 \times 32 \mathrm{~mm}^{2}$ is covered by 1024 pixels. It is surrounded by a $2-\mathrm{D}$ strip readout structure with a pitch of $400 \mu \mathrm{m}$. In total, the active area of $10 \times 10 \mathrm{~cm}^{2}$ is covered using 2048 readout channels. Analogue readout by the APV25S1 ASIC in three sample mode has been chosen in order to profit from amplitude measurements which help to improve the spatial resolution by clustering neighboring hit strips or pixels. A detailed description of the clustering algorithm can be found in [5].

The total amount of material of the detector exposed to the beam corresponds to only $0.4 \%$ of a radiation length $\mathrm{X}_{0}$ or $0.1 \%$ of an interaction length $\lambda_{\mathrm{I}}$.

The signals from the active area are routed to front-end cards mounted at a distance of about $20 \mathrm{~cm}$ from the center by long tracks on the printed circuit board (PCB). For the prototype, the small distance between these tracks of only about $200 \mu \mathrm{m}$ caused crosstalk between neighboring channels in the order of $15 \%$ of the original signal. Although this crosstalk could be eliminated to a large extent by software, the routing of PCB tracks on the final readout foil was modified to maximize the distance between them. Figure 2 shows the difference between the prototype and the final design of the readout foil.

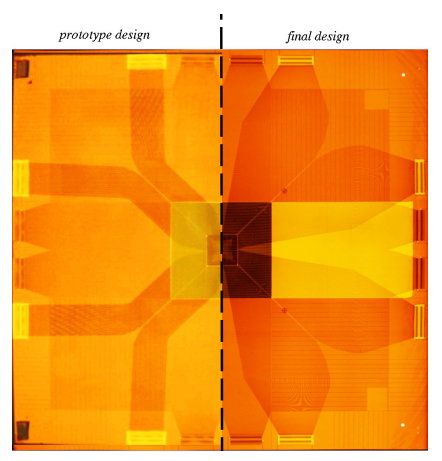

Figure 2. Photos of the readout printed circuit foils on a light table, the prototype design (left hand side) and the final design (right hand side).

The most significant design improvement concerns the use of large area GEM and drift foils, which are coated with $1-2 \mu \mathrm{m}$ copper instead of $5 \mu \mathrm{m}$. This reduces the material budget of the detector by about 30\%, while gain and tracking performance were measured to be on the same level as for the prototype (see below).

\section{Performance of the PixelGEM}

In order to characterize the new detector both the prototype as well as a specimen with the final design were installed in front of the COMPASS silicon beam telescope upstream of the target for a few days in 2006 and 2007, respectively. The tracks used as reference for the analysis are measured by several silicon and scintillating fiber planes and therefore have negligible spatial and temporal uncertainty with respect to the PixelGEM detector. 
To determine the performance of the PixelGEM, data recorded with muon and hadron beams were analyzed. The intensity and flux density of the $160 \mathrm{GeV} / c$ muon beams reached up to $4.8 \cdot 10^{7} / \mathrm{s}$ and $1.2 \cdot 10^{5} \mathrm{~mm}^{-2} \mathrm{~s}^{-1}$ [4], respectively (c.f. Figure 3 (a)). The intensity and flux density of the hadron beam was determined to be $8.5 \cdot 10^{6} / \mathrm{s}$ and $1.6 \cdot 10^{4} \mathrm{~mm}^{-2} \mathrm{~s}^{-1}$ (c.f. Figure 3 (b)).

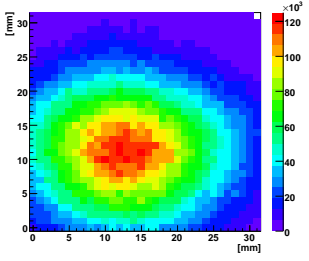

(a) muon beam

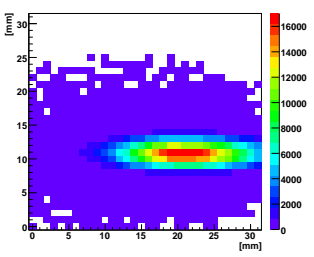

(b) hadron beam
Figure 3. Beam flux density $\left[\mathrm{mm}^{-2} \mathrm{~s}^{-1}\right]$ in the pixel region of the PixelGEM prototype (2006).

In the following, the most important performance characteristics of the pixel region of the PixelGEM tracking detector will be discussed, comparing a low intensity $\left(1.1 \cdot 10^{6} / \mathrm{s}\right)$ and a high intensity muon beam $\left(4.8 \cdot 10^{7} / \mathrm{s}\right)$. The performance of the detector in all analyzed hadron beams matches the one observed for low intensity muon beams and will not be discussed here. A more detailed description of performance studies and assessment under all measured beam conditions (including hadron beams) can be found in [ 4 and [5].

\subsection{Crosstalk suppression}

Analyzing the beam test data, it turned out that the first design of the readout PCB leads to a significant capacitive coupling between pixels, which are routed from the active area to the frontend electronics in adjacent tracks. Due to the complicated pattern, this so called crosstalk does not necessarily affect neighboring pixels.

In order to suppress this source of background during data processing, an algorithm was developed capable of eliminating crosstalk to are large extend (c.f. Figure 4 ).

The algorithm was tuned optimizing the performance of the detector as described in [5]. This tuning also indicates the improvement of the final layout over the prototype readout design.
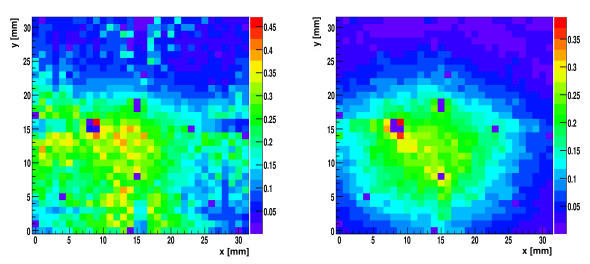

(a) Without crosstalk (b) After crosstalk suppression suppression

Figure 4. Cluster position (pixel region) for low intensity muon beam. The color code is normalized to the number of events and given in percent.

\subsection{Efficiency}

To determine the nominal operation voltage for the detector a voltage scan had been performed (c.f. Figures 5 (a) and (b)). The nominal voltage was chosen to be $3.9 \mathrm{kV}$, where the plateau efficiency of $98.5 \pm 0.1 \%$ for low and $96.4 \pm 0.1 \%$ for high intensity muon beams is reached. All further results mentioned in this publication were obtained with this voltage.

\subsection{Spatial resolution}

By fitting a double Gaussian function with flat background to the residual distributions (c.f. Figures 6 (a) and (b)), the spatial resolution can be determined as the weighted mean of the two components' standard deviations.

The spatial resolution achieved for low intensity muon beams is $90 \mu \mathrm{m}$, while for high intensity muon beams the spatial resolution is increased to $135 \mu \mathrm{m}$. The reason for the decreased performance under high intensity beam conditions is mostly due to pile-up of off-time tracks. 


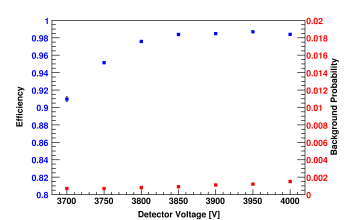

(a) low intensity

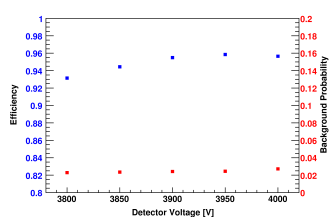

(b) high intensity
Figure 5. Detection efficiency in the pixel region over high voltage settings for muon beam

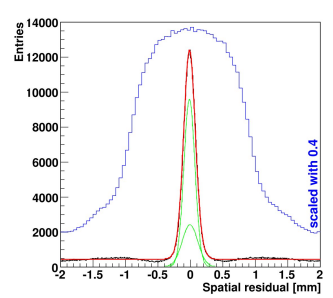

(a) low intensity

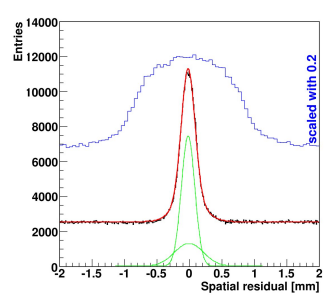

(b) high intensity
Figure 6. Residual distribution in the pixel region (blue: raw hits, black: clusters, red: fit, green: Gaussian components of the fit)

\subsection{Temporal resolution}

The analogue readout of the PixelGEM detector does not allow for a direct time measurement. Thus the track time has to be determined applying pulse shape analysis to the three amplitude samples, which are read out for each event [5]. Figure 7 (a) and (b) show the temporal residual distribution for both low and high intensity muon beams. The temporal resolution is determined to be below $8 \mathrm{~ns}$ for both cases. This can be confirmed off-line, by imposing spatial track selection to suppress the background.

\section{Conclusion}

Being adapted to the special requirements of the hadron program of COMPASS, the novel Pix-

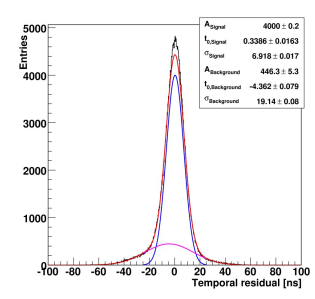

(a) low intensity

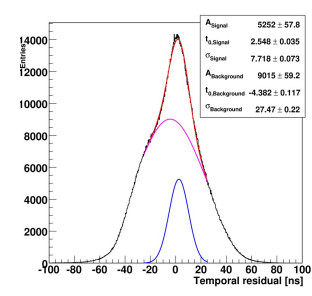

(b) high intensity
Figure 7. Temporal residual distribution of pixel clusters (red: fit, blue: signal, magenta: background)

elGEM detector met the design performance during the beam test in 2006 and 2007. In advance of the 2008 hadron run, the full set of five detectors was installed in the COMPASS spectrometer. Results from the ongoing analysis of the complete PixelGEM central tracking system performance will be available soon.

\section{REFERENCES}

1. P. Abbon et al, The COMPASS Experiment at CERN, Nucl. Instr. Meth. A577 (2007) 455-518

2. C. Altunbas et al, Construction, Test and Commissioning of the Triple-GEM Tracking Detectors for COMPASS, NIM A490 (2002) 177-203

3. B. Ketzer et al, Performance of Triple-GEM Tracking Detectors in the COMPASS Experiment, NIM A535 (2004) 314-318

4. A. Austregesilo, Commissioning and Performance of a Prototype PixelGEM Detector for COMPASS, Technische Universität München, Physik Department E18, diploma thesis (2007)

5. M. Krämer, Performance Optimization of a GEM Detector with Pixel Readout for Beam Tracking in COMPASS, Technische Universität München, Physik Department E18, diploma thesis (2008) 